\title{
Clinical Trial Principal Investigator License
}

National Cancer Institute

\section{Source}

National Cancer Institute. Clinical Trial Principal Investigator License. NCI Thesaurus. Code C115529.

A record of medical and/or other licenses of the principal investigators in a clinical trial. 